\title{
Assessing Microbial Competition in a Hydrogen-Based Membrane Biofilm Reactor (MBfR) Using Multidimensional Modeling
}

\author{
Kelly J. Martin, ${ }^{1,2}$ Cristian Picioreanu, ${ }^{3}$ Robert Nerenberg ${ }^{1}$ \\ ${ }^{1}$ Department of Civil and Environmental Engineering and Earth Sciences, University of \\ Notre Dame, 156 Fitzpatrick Hall, Notre Dame, Indiana 46556 \\ ${ }^{2}$ Department of Civil and Environmental Engineering, University of Michigan, Ann Arbor, \\ Michigan \\ ${ }^{3}$ Department of Biotechnology, Faculty of Applied Sciences, Delft University of \\ Technology, Delft, the Netherlands
}

ABSTRACT: The membrane biofilm reactor (MBfR) is a novel technology that safely delivers hydrogen to the base of a denitrifying biofilm via gas-supplying membranes. While hydrogen is an effective electron donor for denitrifying bacteria (DNB), it also supports sulfate-reducing bacteria (SRB) and methanogens (MET), which consume hydrogen and create undesirable by-products. SRB and MET are only competitive for hydrogen when local nitrate concentrations are low, therefore SRB and MET primarily grow near the base of the biofilm. In an MBfR, hydrogen concentrations are greatest at the base of the biofilm, making SRB and MET more likely to proliferate in an MBfR system than a conventional biofilm reactor. Modeling results showed that because of this, control of the hydrogen concentration via the intramembrane pressure was a key tool for limiting SRB and MET development. Another means is biofilm management, which supported both sloughing and erosive detachment. For the conditions simulated, maintaining thinner biofilms promoted higher denitrification fluxes and limited the presence of SRB and MET. The 2-d modeling showed that periodic biofilm sloughing helped control slow-growing SRB and MET. Moreover, the rough (non-flat) membrane assembly in the $2-\mathrm{d}$ model provided a special niche for SRB and MET that was not represented in the 1-d model. This study compared 1-d and 2-d biofilm model applicability for simulating competition in counter-diffusional biofilms. Although more computationally expensive, the 2 -d model captured important mechanisms unseen in the 1-d model.

Biotechnol. Bioeng. 2015;112: 1843-1853.

(C) 2015 Wiley Periodicals, Inc.

KEYWORDS: MBfR; hollow fiber membrane reactor; denitrification; biofilm model; detachment

Correspondence to: R. Nerenberg

Contract grant sponsor: University of Notre Dame Center for Environmental Science and Technology Bayer Fellowship and NSF project

Contract grant number: CBET0954918

Contract grant sponsor: University of Notre Dame

Received 1 November 2014; Revision received 1 February 2015; Accepted 5 March 2015

Accepted manuscript online 8 April 2015;

Article first published online 7 July 2015 in Wiley Online Library

(http://onlinelibrary.wiley.com/doi/10.1002/bit.25607/abstract).

DOI 10.1002/bit.25607

\section{Introduction}

Hydrogen gas $\left(\mathrm{H}_{2}\right)$ is a clean and efficient electron donor for denitrification in drinking water: it is non-toxic, leaves no organic residual, yields less biosolids, and is inexpensive compared to exogenous organic electron donors (Lee and Rittmann, 2002). However, concern over creating a flammable headspace has limited the use of $\mathrm{H}_{2}$ in full scale water and wastewater treatment technologies (Karanasios et al., 2010). The hollow fiber membrane biofilm reactor (MBfR) offers a safe option for delivering $\mathrm{H}_{2}$ to a biofilm: hollow fibers, sealed at one end, are pressurized with $\mathrm{H}_{2}$ gas, which diffuses through the wall of the membrane to a biofilm that grows on the membrane exterior. This passive delivery scheme allows the biofilm to consume $\mathrm{H}_{2}$ before it enters the bulk liquid.

The hydrogen-based MBfR has been studied extensively for treating nitrate (Celmer-Repin et al., 2010), and other oxidized contaminants, including perchlorate, bromate, chromate, selenite, and arsenate (Chung et al., 2007; Martin and Nerenberg, 2012; Nerenberg and Rittmann, 2004). Hydrogenbased MBfRs for the treatment of nitrate from drinking water have been tested at the pilot scale (Tang et al., 2010; Zhao et al., 2014), and a commercial system was operated in Rancho Cucamonga, California, USA (Martin and Nerenberg, 2012).

In a hydrogen-based, denitrifying MBfR, several undesirable metabolisms may compete with denitrifying bacteria (DNB) for $\mathrm{H}_{2}$ and space within the biofilm, including sulfate-reducing bacteria (SRB) and methanogenic microorganisms (MET). Sulfate and carbon dioxide, common constituents of groundwater, surface water, and wastewater, are reduced by the SRB and MET, respectively. SRB produce sulfide, which is corrosive, odorous, toxic, and can precipitate metals (Widdel, 1988). Furthermore, alkalinity generated by sulfate-reduction may cause the precipitation of hardness or metals on the membrane surface, introducing significant gas transfer resistance and resulting in brittle membranes (Hwang et al., 2009; Van Ginkel et al., 2011). 
MET produce methane, a greenhouse gas with a global warming potential 21 times that of carbon dioxide (Houghton et al., 1996). Understanding how to manage the growth and activity of these microorganisms is important to the performance and life of the reactor.

Studies have shown that DNB effectively outcompete SRB for $\mathrm{H}_{2}$, but due to concentration gradients, $\mathrm{SRB}$ are able to thrive in the inner regions of a biofilm where low nitrate concentrations exist (Tang et al., 2012). Similarly, SRB generally outcompete MET due to kinetic and thermodynamic advantages, yet SRB and MET can coexist in biofilms, even under non-limiting sulfate concentrations (Raskin et al., 1996). In a conventional biofilm, $\mathrm{H}_{2}$ not consumed by DNB becomes available to the SRB and MET residing deep in the biofilm. MBfR biofilms experience counterdiffusion, where $\mathrm{H}_{2}$ is sourced from the base of the biofilm, while nitrate, sulfate, and carbon dioxide enter from the bulk liquid. In this configuration, the inner biofilm sustains low nitrate concentrations and high $\mathrm{H}_{2}$ concentrations, creating favorable environments for SRB and MET. Therefore, the effect and extent of SRB and MET proliferation in the MBfR may differ from conventional understanding and should be systematically studied.

Previous studies on the competition of DNB and SRB in a $\mathrm{H}_{2}$ based MBfR show the onset of sulfate reduction is primarily a function of the substrate loadings: the $\mathrm{H}_{2}$ intramembrane pressure and the loading of nitrate and sulfate by the influent flowrate ( $\mathrm{Lu}$ et al., 2009; Ontiveros-Valencia et al., 2013; Tang et al., 2012; Terada et al., 2006; Ontiveros-Valencia et al., 2012; Xia et al., 2009; Ziv-El and Rittmann, 2009). However, these studies assumed the MBfR biofilm was grown on a flat attachment surface and had uniform thickness. Many biofilms exhibit a heterogeneous morphology, heavily influenced by the fluid dynamics of the reactor (Pavissich et al., 2014). Erosion and prevalence of sloughing events (i.e., detachment of large portions of biofilm) in a stratified biofilm (e.g., SRB in the inner and DNB in the outer regions) may drastically alter the microbial community structure and activity (Elenter et al., 2007; Morgenroth and Wilderer, 2000). Also, past studies never considered the presence of MET, which are of increasing concern in treatment technologies, as methane is a powerful greenhouse gas.

The primary objective of this research was to develop one- and two-dimensional (1-d and 2-d) multispecies biofilm models to study microbial competition of DNB, SRB, and MET in a hydrogen-based, denitrifying MBfR. A 1-d model was used as a baseline to evaluate the effect of biofilm thickness on the microbial community structure and rates of denitrification, sulfate reduction, and methane production. A 2-d particle-based biofilm model, coupled to solution of fluid dynamics and mass transport, was used to further evaluate the effect of detachment on competition between DNB, SRB, and MET in a biofilm with a non-flat attachment surface and a biofilm with complex morphology. Finally, this work compared the suitability of 1-d and 2-d models for studying competition in an MBfR biofilm. The results were used to identify methods to avoid fouling and maintain optimum performance in a counter-diffusional, hydrogen-based system.

\section{Methodology}

\section{One-Dimensional (1-d) Model}

\section{Set-Up}

The 1-d model is constructed in AQUASIM, a water treatment simulation software based on linked reactor compartments (Reichert, 1994). The biofilm reactor compartment includes the biofilm and bulk liquid, coupled by a liquid diffusion layer (LDL). To model the gas-supplying membrane, a completely mixed compartment containing $\mathrm{H}_{2}$ is diffusively linked to the base of the biofilm. Substrate concentrations are assumed constant in the bulk liquid and membrane lumen (gas compartment).

Both the 1-d and 2-d models account for three competing metabolisms: hydrogen-based denitrification, sulfate-reduction, and methanogenesis, according to the balanced redox reactions in Supplementary Information (SI) Table SI. The metabolic processes are simulated according to dual-Monod kinetics with decay (Table I). Denitrification is modeled as a one-step reduction to nitrogen gas, neglecting the formation of intermediates. Sulfidebased denitrification and heterotrophic growth on biodegradable decay products were incorporated into preliminary 1-d models, but growth of these organisms was minimal compared to that of DNB and SRB. Therefore, they are not represented in the model of this study. The biofilm may also foster denitrifying anaerobic methane oxidizing (DAMO) organisms by forming a syntrophic relationship with MET, but DAMO are not considered in this model as their growth rates are much slower than DNB (Chen et al., 2014; Hu et al., 2011). Hydrogen sulfide, a product of sulfate-reduction, can inhibit SRB and MET activity (Hilton and Oleszkiewicz, 1988) and cause the accumulation of DNB intermediates (Sorensen et al., 1980). Inhibition by hydrogen sulfide (Kalyuzhnyi et al., 1998; Levenspiel, 1980), as well as weak acid equilibria (Musvoto et al., 2000; Picioreanu et al., 2010) and inhibition by pH (Angelidaki et al., 1993; Estuardo et al., 2008; Fedorovich et al., 2003), were initially modeled, but showed little effect on the results for the scenarios tested, and are therefore omitted.

The 1-d model solves for concentrations of six soluble state variables-nitrate, sulfate, $\mathrm{H}_{2}$, bicarbonate, methane, and total sulfide ( $\mathrm{HS}^{-}$and $\mathrm{H}_{2} \mathrm{~S}$ ) - and three particulate variables - DNB, SRB, and MET. The stoichiometric matrix, used to relate metabolic processes to state variables, is provided in Table 1. A charge balance is not performed, assuming an excess of counter-ions $\left(\mathrm{Na}^{+}\right.$and $\left.\mathrm{Cl}^{-}\right)$.

Initially, the biofilm thickness $L_{f}$ is $10 \mu \mathrm{m}$, with DNB, SRB, and MET equally distributed. The model disregards biomass attachment during biofilm development. To investigate biofilms of a defined maximum thickness $L_{f, l i m i t}$, the detachment velocity $u_{d e}$ is coupled to biofilm growth velocity $u_{f}$ following the function: $u_{d e}=$ $u_{f}\left(L_{f} / L_{f, \text { limit }}\right)^{10}$ (Matsumoto et al., 2007). With this function, the biofilm grows with almost no detachment until the biofilm thickness reaches $L_{f, \text { limit }}$. At this point, the detachment and growth velocities become equal, which can be interpreted as complete erosion of biomass growth exceeding $L_{f, \text { limit }}$. Modeling the biofilm in this manner allows for evaluation of biofilms thickness, which becomes integral to MBfR performance and differs with biomass management strategies. 
The literature reports kinetic parameters of DNB, SRB, and MET over large ranges, and to our knowledge, no study has estimated the kinetic parameters of the microorganisms of interest using a consistent method. In order to select for parameters without bias of experimental set-up (i.e., inoculum, reactor type, etc.), we calculated the kinetic parameters based on theoretical stoichiometry (Table SI). The maximum specific growth rates $\mu_{\max }$ and yields Yare estimated with the method from Rittmann and McCarty (2001), considering the theoretical portions of electron donor used for energy $f_{e}^{\circ}$. Half-saturation constants $K$ are taken from the literature, and the decay constants $b$, based on limited studies, are assumed equal for each microorganism type. The kinetic parameters are listed in Table SII, and the physical and program parameters used to define the biofilm compartment are provided in Tables SIII and SIV, respectively.

The simulated bulk liquid concentrations are chosen based on values typically found in drinking water sources, including nitrate $S_{\mathrm{NO} 3, b l}\left(0.5-5 \mathrm{~g} \mathrm{~N} \cdot \mathrm{m}^{-3}\right)$, sulfate $S_{S O 4, b l}\left(10-150 \mathrm{~g} \mathrm{SO}_{4}{ }^{2-} \cdot \mathrm{m}^{-3}\right)$, and bicarbonate $S_{\mathrm{HCO}, \mathrm{bl}} \quad\left(300 \mathrm{~g} \mathrm{HCO}_{3}{ }^{-} \cdot \mathrm{m}^{-3}\right)$. The hydrogen sulfide, $\mathrm{H}_{2}$, and methane concentrations are assigned as null. This study evaluates three intramembrane pressures $p_{\mathrm{H} 2}$ between 1.73.7 atm absolute assuming dense membrane with properties provided in Table SIV. A total of 200 days is simulated, which allows for the microbial community and substrate fluxes to reach steady state.

\section{Two-Dimensional (2-d) Model}

The 2-d model consists of a particle-based biofilm submodel, based on mechanistic biomass attachment, growth, spreading, and detachment functions, coupled to a physical submodel providing solution of 2-d fluid dynamics and mass transport. The model is described in detail in Martin et al. (2013) for single-species simulation, therefore only changes made to accommodate multiple species are discussed here. To simulate biofilm growth in an MBfR flow channel, the modeling domain consists of a channel space (width $1.5 \mathrm{~mm}$, length $15 \mathrm{~mm}$ ) with hollow fiber membranes (outer diameter $200 \mu \mathrm{m}$ ) aligned along the bottom boundary to represent a woven membrane fabric, which has been used in pilot and fullscale, spiral-wound MBfR modules (Figure S1) (Martin et al., 2013). To save on computational costs, the biofilm is only grown at the bottom boundary.

\section{Physical Submodel}

Hydrodynamics, mass transport by diffusion and advection, and reaction are solved by finite element analysis in COMSOL 3.5a (Comsol, Stockholm, Sweden, www.comsol.com) (Martin et al., 2013). Steady laminar flow is assumed throughout the channel with average velocities at the inlet ranging from $0.025-0.05 \mathrm{~m} \cdot \mathrm{s}^{-1}$. In regards to mass transfer, the model solves for transport of the same six chemical species as the 1-d model. At each flowrate tested, the average concentration of the chemical species exhibits negligible change between the channel inlet and outlet. The rates and stoichiometry are also the same as for the 1-d model (Table 1). 
Boundary conditions match those of the single-species MBfR model in Martin et al. (2013).

\section{Biofilm Submodel}

The biofilm consists of spherical biomass entities (maximum diameter of $10 \mu \mathrm{m}$ ) that behave according to growth, division, spreading and attachment mechanisms as defined by Picioreanu et al. (2004) and detachment as implemented by Martin et al. (2013). For the multispecies model, each biomass entity is tagged with a species identity (i.e., DNB, SRB, or MET) that is passed on to daughter cells. The three different species are assigned the same biomass density $\rho_{x}$ and maximum diameter $d_{\text {max }}$ (i.e., size at which to divide), but exhibit unique biological characteristics, such as growth and substrate uptake rates. At each time step, the number of particles that randomly attach to the outer biofilm and/or exposed membrane is made proportional $(0.5 \%)$ to the number of particles for that given species. This differs from Martin et al. (2013), where the number of entities to attach at each time step is assigned by the user and remains constant. We implemented this change so the number of attached particles is proportional to the community size, as the number of DNB, SRB, and MET biomass entities can differ by orders of magnitude. The detachment mechanism, based on internal stress due to hydraulic shear, simulates both erosion and sloughing. When internal stress exceeds a defined maximum value $\sigma_{d e}$, the biomass entity is removed from the model (Picioreanu et al., 2000). The biofilm submodel and main program are written in MATLAB R2009b (Mathworks, Natick, Massachusetts, www.mathworks.com) and linked to COMSOL 3.5a.

\section{Parameters and Simulations for 2-d Model}

Table SV provides the values used specifically in the 2- $\mathrm{d}$ model. The processes and stoichiometry (Table I), kinetic parameters (Table SII), and physical parameters (Table SIII) are identical to those of the 1-d model. Discussion on matching biomass density $\rho_{x}$ and effective diffusivity between the 1- $\mathrm{d}$ and 2-d models is provided in Section S2 of the SI.

Simulations with inlet velocities of 0.025 and $0.04 \mathrm{~m} \cdot \mathrm{s}^{-1}$ were conducted with inlet substrate concentrations of the bulk liquid substrates and the intramembrane $\mathrm{H}_{2}$ pressure held at based case conditions. Biofilm development over 40 days was computed, with a time step $\delta t$ of $4 \mathrm{~h}$. Simulations ran on a workstation with a $3 \mathrm{GHz} 8$ core processor with 32 GB RAM. Simulation times approximately 8 days, with intermediate files saved at each time step.

\section{Results and Discussion}

\section{One-Dimensional Modeling}

\section{Effect of Substrate Concentrations}

Both the 1-d and 2-d models were assigned base case bulk liquid/ inlet concentrations of nitrate $S_{\mathrm{NO}, b l}=1 \mathrm{~g} \mathrm{~N} \cdot \mathrm{m}^{-3}$, sulfate $S_{S O 4}$, ${ }_{b l}=75 \mathrm{~g} \mathrm{SO}^{2-} \cdot \mathrm{m}^{-3}$ and bicarbonate $S_{\mathrm{HCO}, b l}=300 \mathrm{~g} \mathrm{HCO}_{3}{ }^{-} \cdot \mathrm{m}^{-3}$, with null methane, $\mathrm{H}_{2}$, and total sulfides. The base case intramembrane $\mathrm{H}_{2}$ pressure was maintained at 2.7 atm absolute, a)
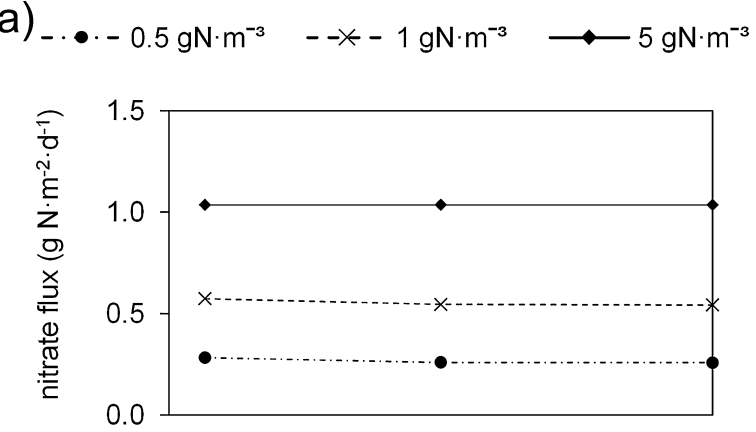

b)
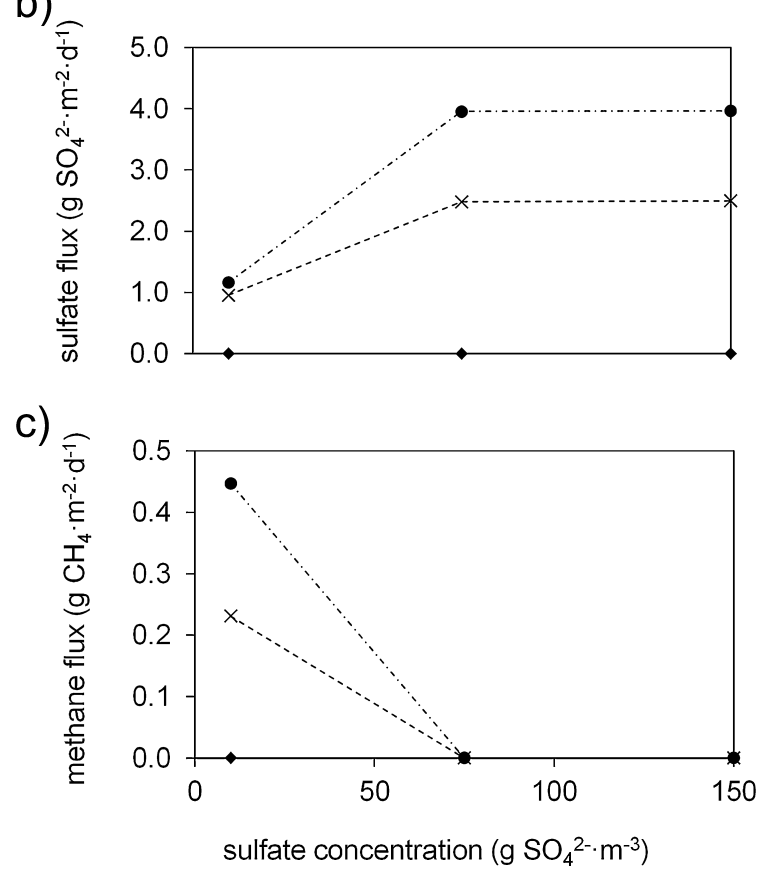

Figure 1. a) Nitrate flux, b) sulfate flux, and c) methane flux for variable bulk liquid sulfate and nitrate concentrations.

and the biofilm thickness was limited to $200 \mu \mathrm{m}$ (for the 1-d model only). In the following sections, the simulation variables are only designated when they differ from the base case scenario.

Figure 1 shows the nitrate, sulfate, and methane fluxes for the bulk liquid nitrate and sulfate concentrations tested. Experimental hydrogen-based MBfRs treating nitrate in drinking water have demonstrated rates of denitrification between $0.6-6.5 \mathrm{~g} \mathrm{~N} \cdot \mathrm{m}^{-2}$ $\cdot \mathrm{d}^{-1}$ (Adham et al., 2006; Celmer-Repin et al., 2010; Terada et al., 2006). In addition, Ontiveros-Valencia et al. (2012) experimentally quantified nitrate fluxes between $0.02-1.3 \mathrm{~g} \mathrm{~N} \cdot \mathrm{m}^{-2} \cdot \mathrm{d}^{-1}$ and sulfate fluxes between $0-2.8 \mathrm{~g} \mathrm{SO}_{4}{ }^{2-} \cdot \mathrm{m}^{-2} \cdot \mathrm{d}^{-1}$ for a hydrogenbased MBfR operated under a large range of substrate conditions. The modeled flux results compared reasonably well to the experimental values in terms of magnitude, but should not be compared directly as membrane type, module design, and operational conditions varied widely among the studies.

In accordance with the literature, the 1-d modeling results showed DNB to effectively outcompete SRB and MET (OntiverosValencia et al., 2012; Tang et al., 2012; Ziv-El and Rittmann, 2009). 


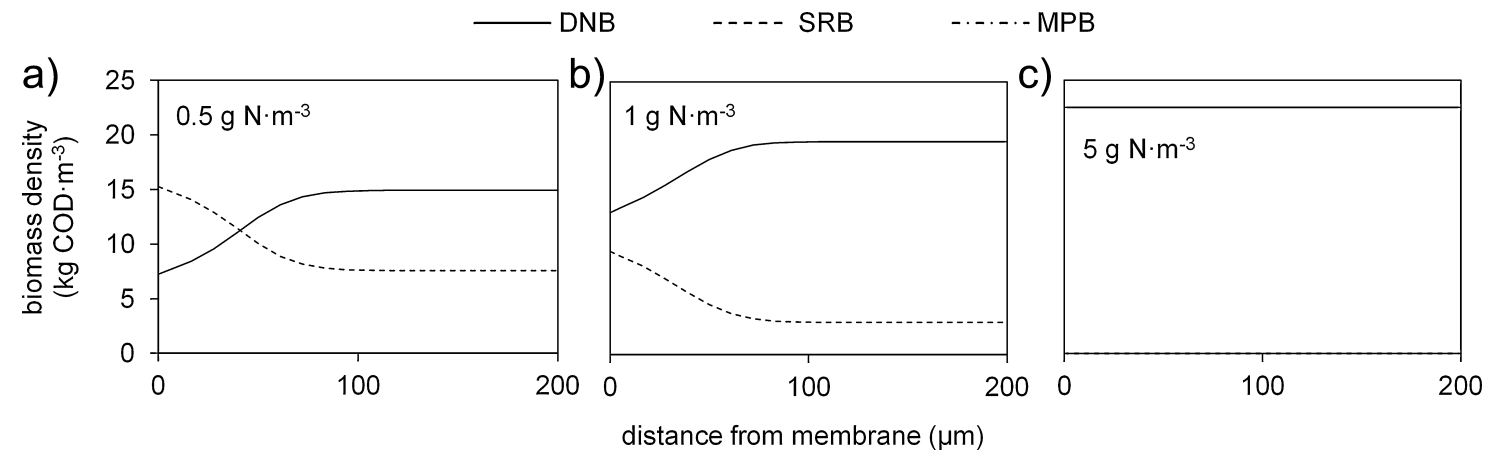

Figure 2. Steady state microbial community structure of a $200 \mu \mathrm{m}$ thick biofilm for three different bulk liquid nitrate concentrations: a) $\left.0.5 \mathrm{~g} \mathrm{~N} \cdot \mathrm{m}^{-3}, \mathrm{~b}\right) 1 \mathrm{~g} \mathrm{~N} \cdot \mathrm{m}^{-3}$, and c) $5 \mathrm{~g}$ $\mathrm{N} \cdot \mathrm{m}^{-3}$. The sulfate concentration was $75 \mathrm{~g} \mathrm{SO}{ }^{2-} \cdot \mathrm{m}^{-3}$.

Nitrate flux went unaffected by bulk sulfate concentrations between 10 and $150 \mathrm{~g} \cdot \mathrm{m}^{-3}$ (Fig. 1a), while increasing $S_{N O 3, b l}$ from 0.5 to $5 \mathrm{~g}$ $\mathrm{N} \cdot \mathrm{m}^{-3}$ negatively impacted sulfate and methane fluxes (Fig. lb,c). At $5 \mathrm{~g} \mathrm{~N} \cdot \mathrm{m}^{-3}$, DNB dominated the entire biofilm (Fig. 2c), resulting in minimal sulfide and methane production. At lower bulk liquid nitrate concentrations, SRB proliferated greatest near the membrane (Fig. 2a,b), where relatively low nitrate and high $\mathrm{H}_{2}$ concentrations existed. Sulfate fluxes experienced negligible change from the increase in the bulk liquid sulfate concentration from 75 to $150 \mathrm{~g} \mathrm{SO}_{4}{ }^{2-} \cdot \mathrm{m}^{-3}$ (Fig. 1b). Sulfate concentrations within the biofilm greatly exceeded the SRB half-saturation constant $K_{S O 4}$ $=2.7 \mathrm{~g} \mathrm{SO}_{4}{ }^{2-} \cdot \mathrm{m}^{-3}$, meaning sulfate was never rate-limiting. Only at both low nitrate $\left(0.5 \mathrm{~g} \mathrm{~N} \cdot \mathrm{m}^{-3}\right)$ and sulfate concentrations $(10 \mathrm{~g}$ $\mathrm{SO}_{4}{ }^{2-} \cdot \mathrm{m}^{-3}$ ) (Fig. 3) did MET populations become established and were methane fluxes observed (Fig. 1c). SRB and MET proliferated best near the membrane (biofilm base), leading to the greatest total sulfide $\left(\mathrm{H}_{2} \mathrm{~S}+\mathrm{HS}^{-}\right)$(Fig. 3c) and methane (Fig. 3d) concentrations in this region. Past modeling and experimental research suggests that MBfRs are susceptible to competition from SRBs, especially when used to achieve low effluent nitrate concentrations (Lu et al., 2009; Tang et al., 2012). The results of this study are consistent with these findings, and show that MET may also be a concern.

Experimental research has also stressed the importance of controlling the $\mathrm{H}_{2}$ supply: at relatively low $\mathrm{H}_{2}$ intramembrane a)

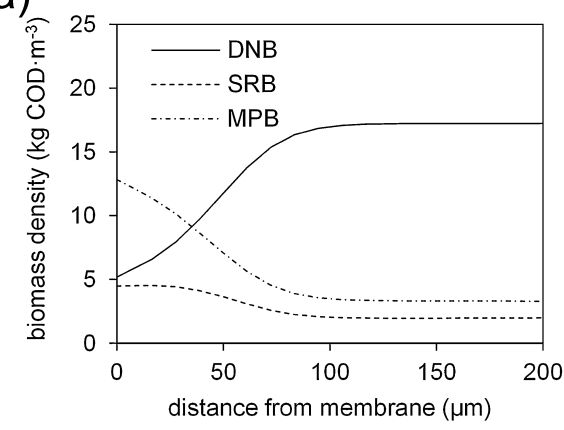

c)

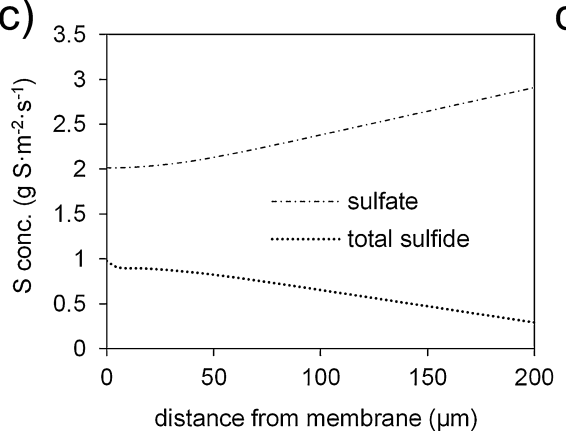

b)

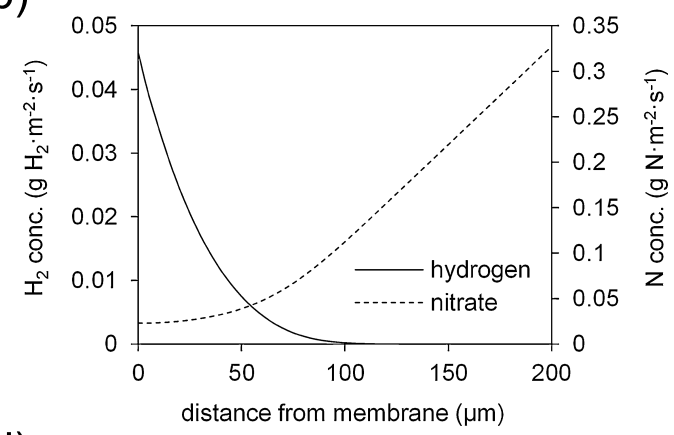

d)

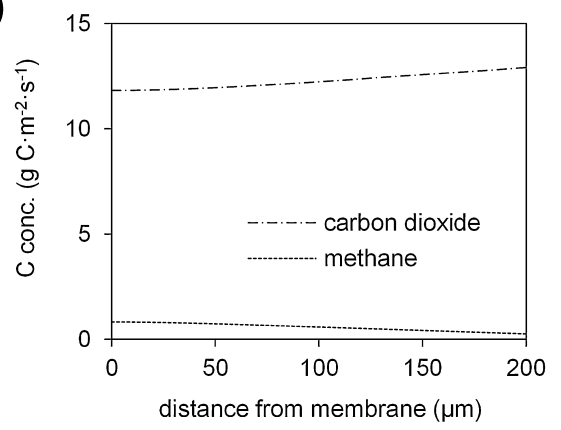

Figure 3. Distribution of a) biomass fractions, b) hydrogen and nitrogen, c) sulfate and total sulfide, and d) methane and carbon dioxide for a $200 \mu \mathrm{m}$ thick biofilm. The bulk liquid nitrogen concentration was $0.5 \mathrm{~g} \mathrm{~N} \cdot \mathrm{m}^{-3}$ and sulfate concentrations $\left(10 \mathrm{~g} \mathrm{SO}_{4}{ }^{2-} \cdot \mathrm{m}^{-3}\right.$ or $\left.3.3 \mathrm{~g} \mathrm{~S} \cdot \mathrm{m}^{-3}\right)$. 
pressures, denitrification is solely supported, but may be ratelimited by the $\mathrm{H}_{2}$ flux. By increasing the $\mathrm{H}_{2}$ pressure, a point will be reached where the onset of sulfate reduction occurs. Raising the $\mathrm{H}_{2}$ pressure past this onset point results in a large gain in sulfate reduction, but little change in the rate of denitrification (OntiverosValencia et al., 2012,2014; Tang et al., 2012). We explored the effect of intramembrane pressure (Section S3) and the results agreed with the aforementioned studies, as excess $\mathrm{H}_{2}$ led to unwanted growth of SRB and MET.

\section{Effect of Biofilm Thickness}

This study evaluated the steady-state nitrate and sulfate fluxes obtained at three biofilm thicknesses: 100, 200, and $300 \mu \mathrm{m}$ (Fig. 4). For $S_{N O 3, b l}=5 \mathrm{~g} \mathrm{~N} \cdot \mathrm{m}^{-3}$, DNB dominated the biofilm at each thickness tested, while sulfate flux was null. However, at lower nitrate concentrations, SRB and MET established themselves in the biofilm as evidenced by the increase in sulfate reduction and methane production. Interestingly, biofilm thickness was detrimental to the nitrate flux (Fig. 4a), but favorable to sulfate flux (Fig. 4b). This behavior is unique to counter-diffusional biofilms and can be attributed to dual-substrate limitation, where the biofilm near the membrane is nitrate-limited and the biofilm near the bulk liquid is hydrogen-limited. For thicker biofilms, nitrate encountered greater diffusional resistance to reach the inner biofilm regions containing $\mathrm{H}_{2}$, which effectively lowered the nitrate flux. Consequently, DNB experienced lower growth rates near the membrane, where a greater percentage of $\mathrm{H}_{2}$ was available to support sulfate-reducing and methanogenic growth and activity (Fig. S3). Due to relatively large concentrations of sulfate and bicarbonate in the bulk liquid, increased diffusional resistance to the mass transport of these substrates imparted no change on their fluxes. As demonstrated by the results in Section S5, LDL thickness (e.g., due to slow flow or low mixing intensity) also served as a source of diffusional resistance and exhibited a similar effect as biofilm thickness by lowering the DNB activity and improving the competitiveness of SRB and MET.

\section{Two-Dimensional Modeling}

The evolution of a 2-d biofilm structure is pictorially presented in Figure 5 for base case substrate conditions and an average flow velocity of $u=0.25 \mathrm{~m} \cdot \mathrm{s}^{-1}$. An animation of the simulated biofilm development is provided in the SI (Movie S1). As expected, the faster-growing DNB quickly established themselves (Day 10), but in time, slower-growing SRB and MET populations increased at the biofilm base. Excessive growth of DNB from Day 15 to Day 30 resulted in a decline in nitrate consumption and an increase in sulfate and methane formation (Fig. 6) due to excessive biofilm thickness, as also observed in the 1-d case. Assuming a constant flowrate at the inlet, an increase in biofilm thickness caused constriction in the flow channel, and consequently, increased shear stress exerted on the biofilm. This led to increased biomass detachment through both erosion and sloughing (i.e., detachment of large pieces of biomass) mechanisms. On Day 32, a major sloughing event detached a large biofilm section containing DNB, SRB, and MET, as outlined in Figure 5, and a sharp decrease in total
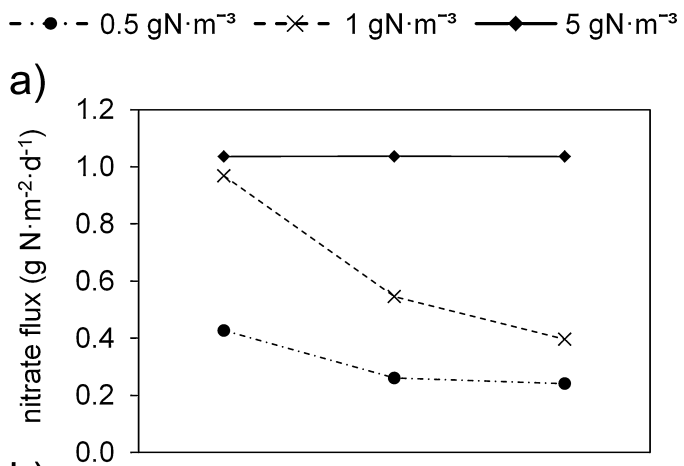

b)

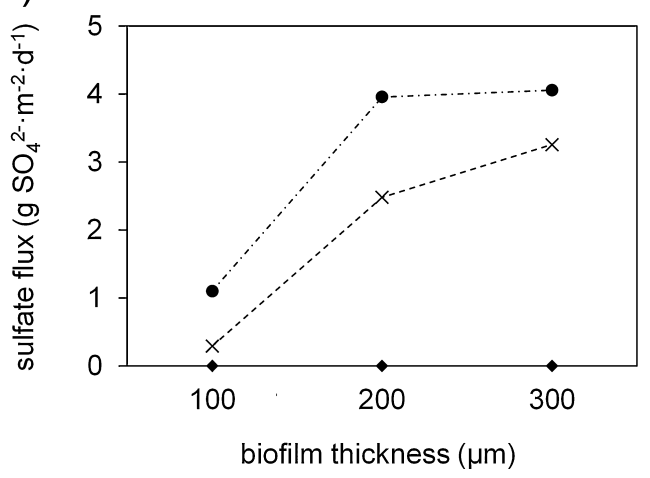

Figure 4. Effect of biofilm thickness on: a) nitrate flux and b) sulfate flux as the bulk liquid nitrate concentration varied. For each scenario, the bulk liquid sulfate concentration was $75 \mathrm{~g} \mathrm{SO}_{4}{ }^{2-} \cdot \mathrm{m}^{-3}$. Methane fluxes were zero.

biomass (Fig. 6). By Day 40, the region was re-colonized and dominated by faster-growing DNB. A series of detachment events from Day 30 to Day 40 led to improvements in nitrate flux and a drop in sulfate and methane fluxes (Fig. 6). Frequent sloughing events essentially kept the biofilm "young" and therefore, favored faster-growing DNB.

Figure 7 depicts 2-d substrate distributions for the section of biofilm existing between 9.5 and $12 \mathrm{~mm}$ in the Figure 5 channel. As the biofilm grew in thickness, $\mathrm{H}_{2}$ became limiting, being available only near the membrane surface. For thick biofilms, nitrate had to diffuse into the middle of the biofilm to be consumed. Over time, the SRB and MET populations increased, with microcolonies observable by pockets of lower sulfate or greater methane concentrations. Between Days 30 and 40, sloughing and regrowth decreased SRB and MET populations. Also note on Day 20, the decreased presence of SRB and MET in the thin biofilm section followed a sloughing event and corresponded to a decline in methane production.

\section{Comparison of 1-d and 2-d Modeling Results}

This section compares 2-d modeling results, where the biofilm experienced an average steady-state thickness of $300 \mu \mathrm{m}$ (with fluctuations between 250 and $350 \mu \mathrm{m}$, obtained at $u=0.04 \mathrm{~m} \cdot \mathrm{s}^{-1}$ ), and two steady-state 1-d simulations for a $300 \mu \mathrm{m}$ thick biofilm. For the first 1-d simulation (1-d FM), a flat membrane, per base case conditions, was assumed. The second 1-d simulation (1-d HFM) 


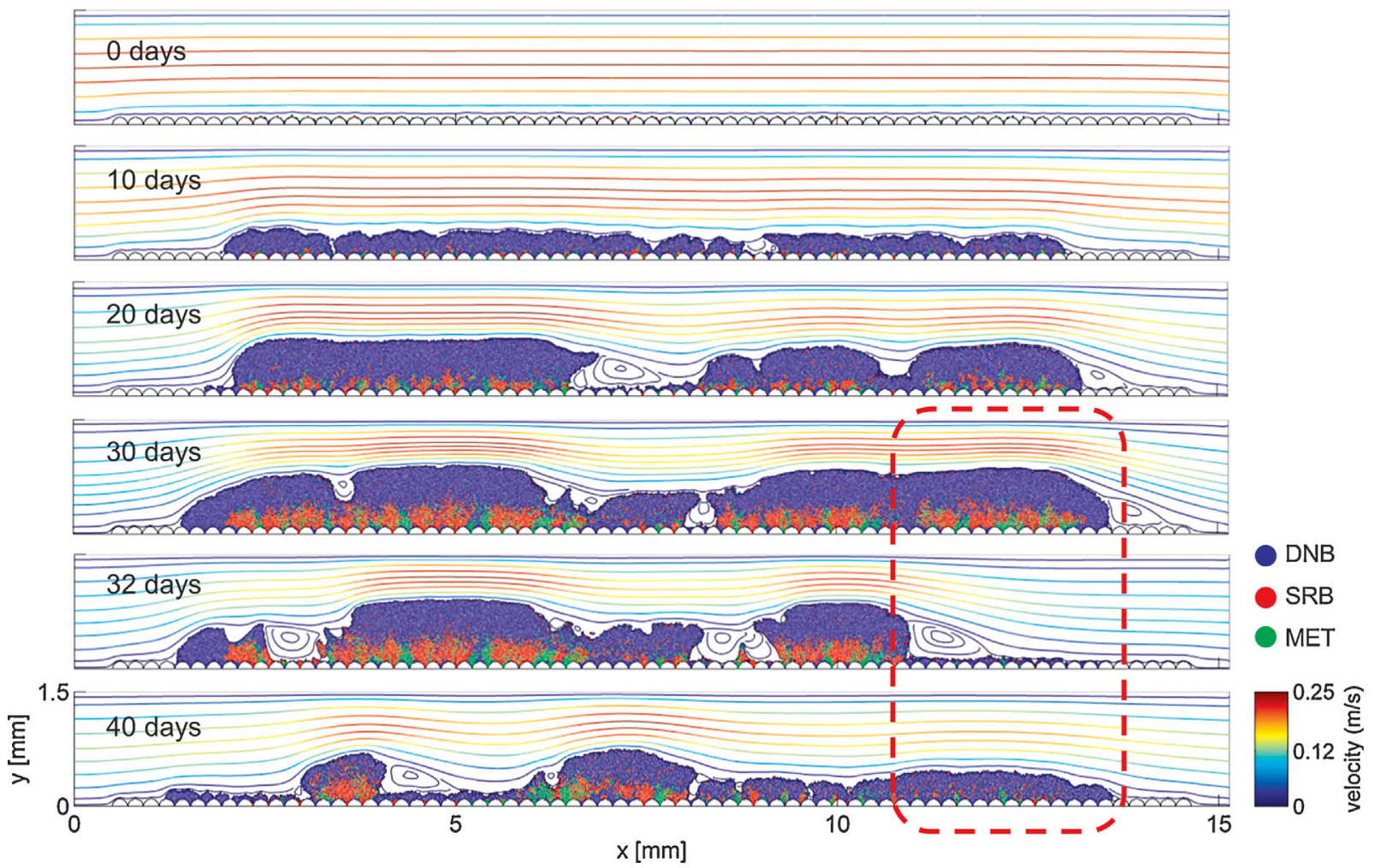

Figure 5. Evolution of microbial community structure for an average flowrate of $0.025 \mathrm{~m} \cdot \mathrm{s}^{-1}$. A major sloughing event occurred between Day 30 and 32 , as outlined in red. To view the figure in color, please refer to the electronic version of the article.

treated the attachment surface as hollow fiber membrane fabric by increasing the ratio of inner biofilm area (i.e., hydrogen-supplying membrane) to outer biofilm area. To elaborate, a flat attachment surface has a $A_{\text {inner: }}: A_{\text {outer }}$ ratio of 1:1, whereas the membrane fabric ratio equals $\pi / 2: 1$ due to membrane circularity.

Figure 8a-c compares total specific biomass concentration (i.e., mass per projected area) for the 1-d and 2-d biofilms between Days $0-40$. The 2 -d results in Figure 8 are based on a single simulation, in which a degree of randomness determined the solution (i.e., due to biomass attachment placement, chosen direction for biomass division, etc.) (Martin et al., 2013). Frequent sloughing events in the 2-d model can make comparisons between the 1-d and 2-d results difficult, but in spite of the inherent stochastic events, we draw some general conclusions from the assessment, as described below.

All three models resulted in fairly similar total specific DNB concentrations (Fig. 8). DNB were the fastest growing organisms and could outcompete SRB and MET for resources and space in the biofilm. Accounting for the increased HFM area enhanced the $\mathrm{H}_{2}$ supply, favoring SRB and MET. Therefore, the 1-d HFM model better matched the 2-d modeling results for the SRB and MET.

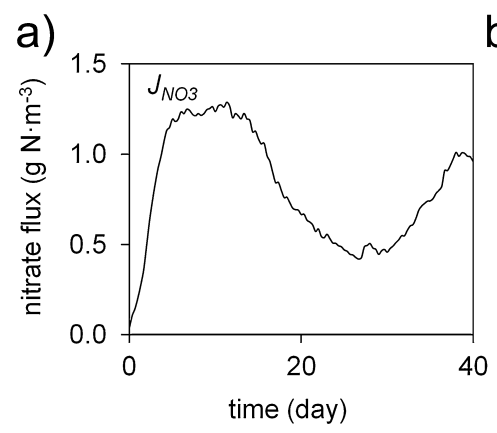

b)

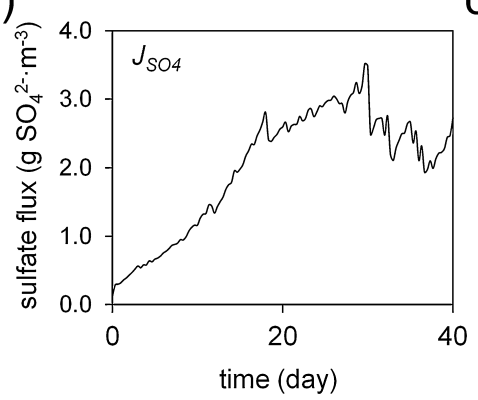

c)

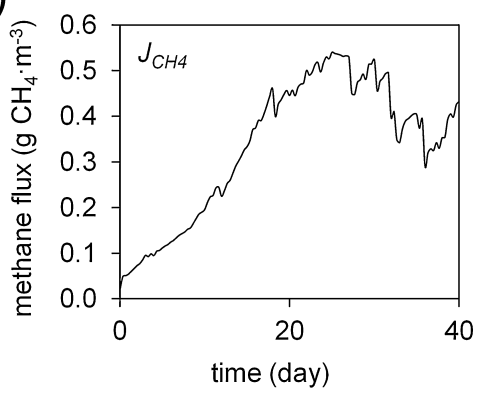

Figure 6. Overall a) nitrate, b) sulfate, and c) methane fluxes for a $2-\mathrm{d}$ model simulation where average channel velocity $=0.25 \mathrm{~m} / \mathrm{s}$. 

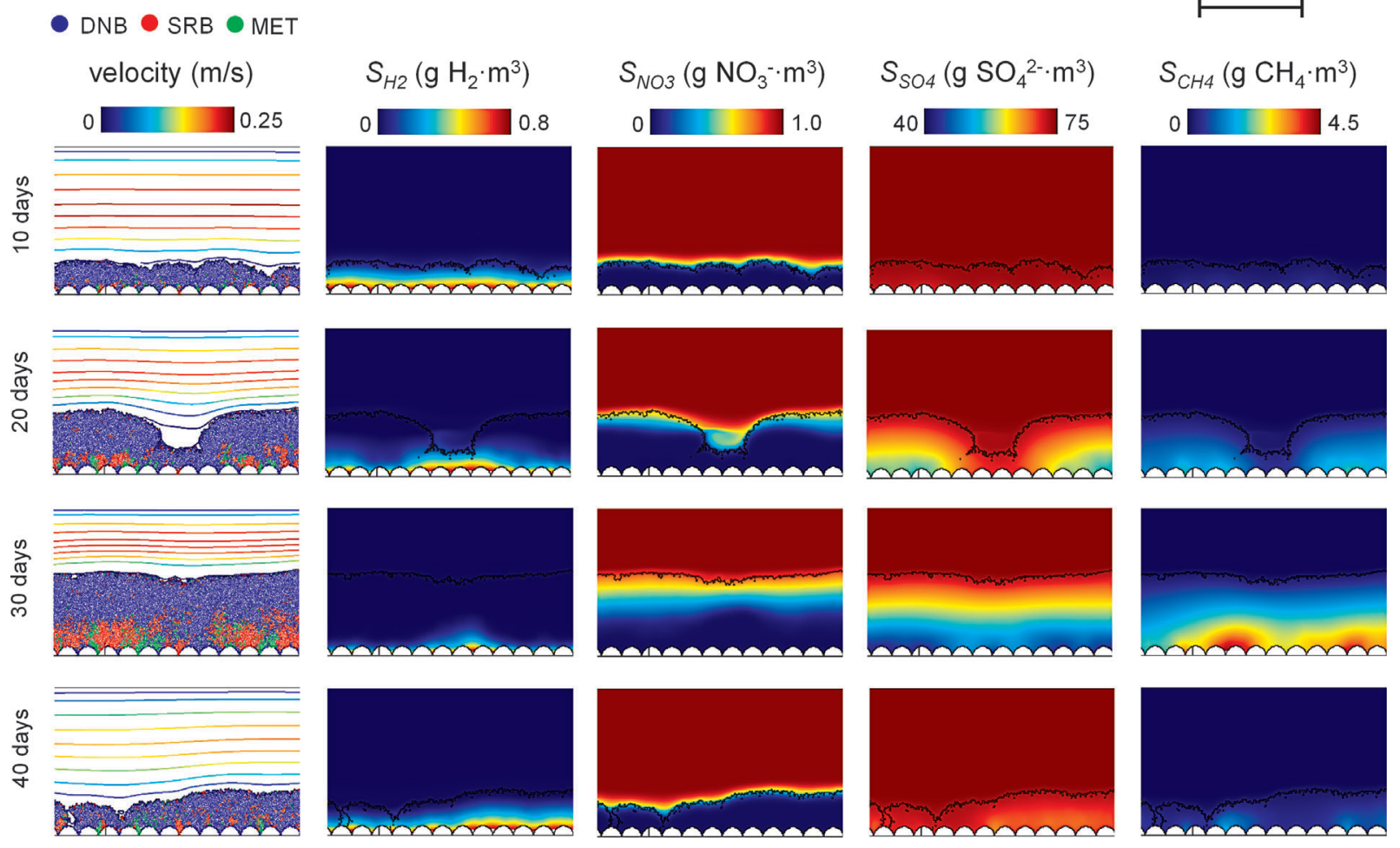

Figure 7. Changes in substrate distributions in time for a section of biofilm. To view the figure in color, please refer to the electronic version of the article.

However, the 1-d HFM and 2-d results for MET development diverged around Day 20. While the 2-d model predicted the MET population to increase, the 1-d model MET population was eventually outcompeted by SRB.

In the 1-d model, DNB, SRB, and MET were assumed to be wellmixed within any given small biofilm volume. The MET faced competition from SRB and DNB simultaneously, which, in the long term, led to complete loss of MET in the 1-d models. In contrast, the 2- $\mathrm{d}$ model accounted for niches between the circular membrane fibers. The niches spatially separated randomly seeded SRB and MET, so that pockets of MET thrived where SRB were absent, as visible in Figure 9. The populations seeded in these niches had an advantage due to high $\mathrm{H}_{2}$ concentrations, low nitrate concentrations (for a developed biofilm), and protection from detachment. Upon a sloughing incident, most biomass was lost, but microbes in the niches remained due to protection from the fibers.

The 1-d and 2-d substrate fluxes agreed for the first 10 days (without sloughing events), but experienced important differences later (Fig. 8d-e), where the 2-d model predicted higher nitrate flux, but lower sulfate flux than the 1-d HFM model. The location of SRB within the 2-d biofilm was at least partially responsible for this discrepancy. During their transport within the growing biofilm, SRB moved vertically (i.e., away from the membrane), as well as horizontally, forming "microcolonies" (Fig. 9). In the 1-d model, a large percentage of the SRB population resided near the membrane where $\mathrm{H}_{2}$ concentrations were greatest, and this stratification was uniform across the membrane surface. The 2-d model also accounted for variable thickness, local concentration boundary layer, and area enlargement, which contributed to the difference. Finally, the 1-d and 2-d models supported similar methane fluxes up to day 20, after which, the METwere driven to extinction in the 1-d but not in the 2-d model, following the previously discussed pattern.

Selecting between the 1-d and 2-d model depends on user knowledge, computational resources, and the nature of the question. Setting-up, operating, and calibrating a 2-d model is more difficult than a 1-d model, yet the 2-d model has the distinct ability to answer questions on biofilm structure, the clustered distribution of microbial types, and the effect of detachment. As this study demonstrated, the geometry of the MBfR substratum is critical to the structure and function of a counter-diffusional biofilm. Modeling in 2-d may be more pertinent to representation of counter-diffusional biofilms than conventional biofilms with inert attachment surfaces. The 2-d geometry not only determines the surface area available to the supply of the gaseous substrate, but also creates niches that support slower growing microorganisms due to greater concentrations of gaseous substrate and protection from shear.

\section{Implications for Practice and Research Opportunities}

The results presented are specific to the model definition and scenarios tested. However, the trends provide general guidelines for minimizing SRB and MET proliferation in a hydrogen-based MBfR. 


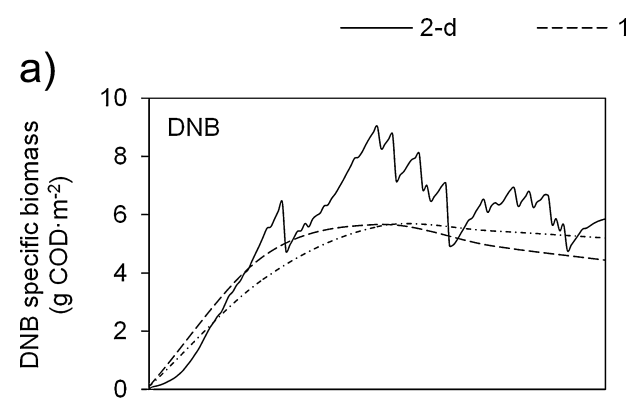

-d HFM $\quad$-......... 1-d flat mem.

b)

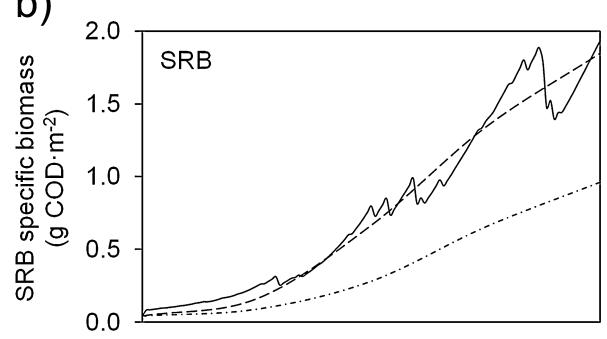

d)

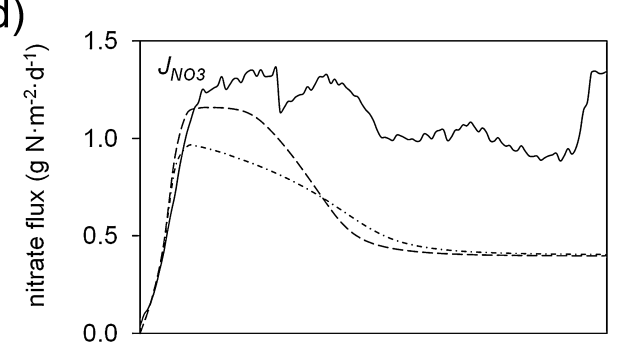

e)

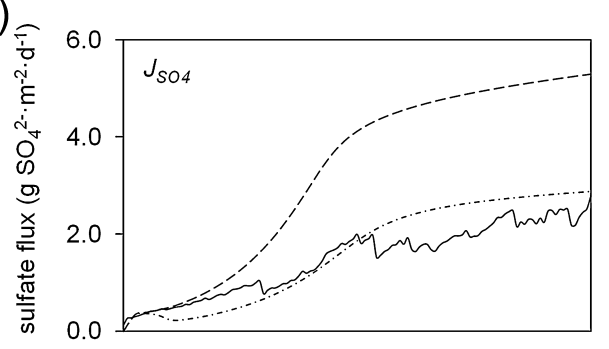

C)
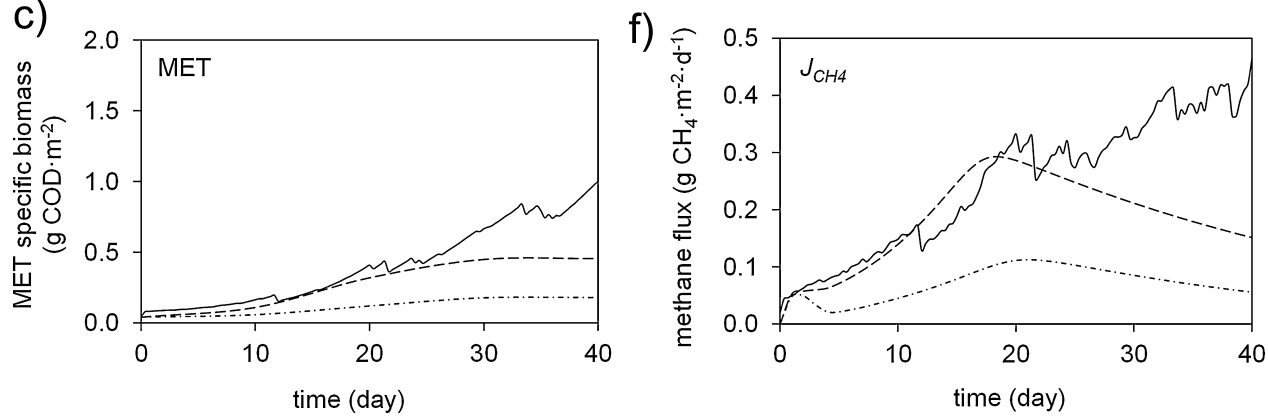

Figure 8. Comparison of biomass growth in the 1-d and 2-d models for a) DNB, b) SRB, and c) MET. Comparison of the d) nitrate, e) sulfate, and f) methane fluxes for the 1-d and 2-d models.

Low nitrate concentrations are a requirement for SRB and MET proliferation, and denitrification and diffusional resistance are responsible for the nitrate concentration gradient within the biofilm. Assuming the effluent nitrate concentration is set, an MBfR operator has control over the rate of denitrification and extent of diffusional resistance through adjustment of the intramembrane $\mathrm{H}_{2}$ pressure and the reactor hydraulic regime, which influences the biofilm thickness, LDL thickness, and detachment, to some extent.
There exists an acceptable range of operational $\mathrm{H}_{2}$ pressures, where denitrification is not significantly limited, yet SRB and MET growth is not excessive. The appropriate $\mathrm{H}_{2}$ intramembrane pressure setpoint may be dynamic as the reactor starts-up or loading rates vary. Research should be conducted on the correlation between $\mathrm{H}_{2}$ intramembrane pressure and performance parameters in order to design a feedback loop control strategy. Another important consideration for full scale systems with sealed end hollow fibers is
22 days
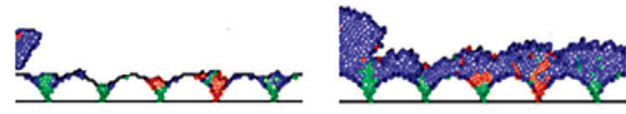

33 days

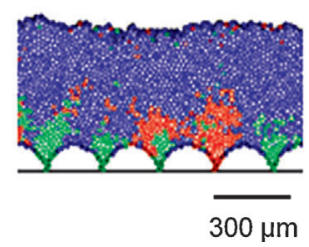

- DNB

SRB

MET

Figure 9. Niches between hollow fibers help to seed the reactor with slow-growing SRB and MET. To view the figure in color, please refer to the electronic version of the article. 
gas back-diffusion (Ahmed and Semmens, 1992; Martin, 2013). Gases from the biofilm (e.g., nitrogen and carbon dioxide) diffuse into the lumen of the membrane and collect near the plugged end, creating a $\mathrm{H}_{2}$ partial pressure gradient along the length of the fiber (i.e., $\mathrm{H}_{2}$ supply may significantly decrease with distance from the inlet). The effect of gas back-diffusion on performance and methods to prevent or accommodate the resulting biofilm spatial heterogeneity are topics that requires more study.

For the conditions simulated, relatively thick biofilms and LDLs negatively impacted denitrification and allowed for greater rates of sulfate reduction and methanogenesis. By far the most commonly practiced strategies for managing biofilm thickness and density are intermittent periods of high shear and/or gas sparging, with other approaches including chemical treatment, temperature, or physical impact by fluidized plastic media (Martin and Nerenberg, 2012). Reactor design and membrane configuration also prove important for the fluid dynamics and detachment, as previously evaluated in Martin et al. (2013).

Conventionally, erosion is considered favorable for maintaining an active biofilm, while sloughing is avoided to prevent exposed membrane. However, SRB and MET can be difficult to remove once established at the inner regions of the biofilm. SRB have been demonstrated to persist in an experimental MBfR biofilm, even during periods of low sulfate-reducing activity (Ontiveros-Valencia et al., 2012). Sloughing events can remove the SRB and MET, though reduced fluxes and/or $\mathrm{H}_{2}$ export to the bulk liquid may lead to undesirable consequences. More research is needed on the implications of sloughing, where issues such as clogging or the formation of preferential flow zones are addressed. In addition, MBfR reactor hydrodynamics and the promotion of detachment events with laminar and turbulent flow regimes requires further study. Fast flowrates and turbulent flows were not modeled in this study due to the computational expense, however hydraulic cleaning has been evaluated by a previous 2-d modeling study for controlling biofouling in a reverse osmosis unit (Radu et al., 2012).

\section{Conclusions}

SRB and MET may proliferate in a denitrifying MBfR, consuming $\mathrm{H}_{2}$ and producing undesirable byproducts.

- High $\mathrm{H}_{2}$ concentrations, low nitrate concentrations, thick biofilms, and thick liquid diffusion layers are favorable to SRB and MET.

- MET are outcompeted by DNB and SRB, unless nitrate and sulfate concentrations are low.

- Avoiding high $\mathrm{H}_{2}$ concentration in the biofilm, by controlling the $\mathrm{H}_{2}$ supply pressure can control proliferation of SRB and MET.

- A rough (non-flat) membrane can promote thriving of SRB and MET by providing niches with high $\mathrm{H}_{2}$ concentrations, low nitrate concentration, and shelter from detachment.

- Periodic biofilm sloughing can help control SRB and MET.

- The 2-d model allowed novel mechanisms, not previously described for MBfRs, to be captured.

We acknowledge the University of Notre Dame Center for Research Computing for use of the computing cluster. K. J. Martin was funded by the
University of Notre Dame Center for Environmental Science and Technology Bayer Fellowship and NSF project CBET0954918 (Nerenberg CAREER award). C. Picioreanu was supported by a Melchor Visiting Professor grant from University of Notre Dame.

\section{References}

Adham S, Gillogly T, Lehman G, Rittmann BE, Nerenberg R. 2006. Membrane Biofilm Reactor Process for Nitrate and Perchlorate Removal. Denver, CO: AWWA Research Foundation.

Ahmed T, Semmens MJ. 1992. The use of independently sealed microporous hollow fiber membranes for oxygenation of water: Model development. J Mem Sci 69:11-20.

Angelidaki I, Ellegaard L, Ahring BK. 1993. A mathematical model for dynamic simulation of anaerobic-digestion of complex substrates: Focusing on ammonia inhibition. Biotechnol Bioeng 42(2):159-166.

Celmer-Repin D, Hwang JH, Cicek N, Oleszkiewicz JA. 2010. Autotrophic nitrogenremoving biofilms on porous and non-porous membranes Environ. Technol 31(12):1391-1401.

Chen X, Guo J, Shi Y, Hu S, Yuan Z, Ni BJ. 2014. Modeling of simultaneous anaerobic methane and ammonium oxidation in a membrane biofilm reactor. Env Sci Technol 48:9540-9547.

Chung J, Rittmann BE, Wright WF, Bowman RH. 2007. Simultaneous bio-reduction of nitrate, perchlorate, selenate, chromate, arsenate, and dibromochloropropane using a hydrogen-based membrane biofilm reactor. Biodegradation 18:199-209.

Elenter D, Milferstedt K, Zhang W, Hausner M, Morgenroth E. 2007. Influence of detachment on substrate removal and microbial ecology in a heterotrophic/ autotrophic biofilm. Water Res 41:4657-4671.

Estuardo C, Marti MC, Huilinir C, Aspe E, von Bennewitz MR. 2008. Improvement of nitrate and nitrite reduction rates prediction. Electron J Biotechn 11(3): 73-82.

Fedorovich V, Lens P, Kalyuzhnyi S. 2003. Extension of anaerobic digestion model No. 1 with processes of sulfate reduction. Appl Biochem Biotechnol 109(1-3):33-45.

Hilton BL, Oleszkiewicz JA. 1988. Sulfide-induced inhibition of anaerobic digestion. J Environ Eng 114(6):1377-1391.

Houghton JT, Meira Filho LG, Callander BA, Harris N, Kattenberg A, Maskell K. 1996. Climate change 1995. the science of climate change. Summary for policymakers and technical summary of the working group I report. Cambridge, United Kingdom: Cambridge University Press.

Hu S, Zeng RJ, Keller J, Lant PA, Yuan Z. 2011. Effect of nitrate and nitrite on the selection of microorganisms in the denitrifying anaerobic methane oxidation process. Environ Microbiol Rep 3(3):315-319.

Hwang JH, Cicek N, Oleszkiewicz J. 2009. Inorganic precipitation during autotrophic denitrification under various operating conditions. Environ Technol 30(13):1475-1485.

Kalyuzhnyi SV, Fedorovich V, Lens P, Po LH, Lettinga G. 1998. Mathematical modelling as a tool to study population dynamics between sulfate reducing and methanogenic bacteria. Biodegradation 9:187-199.

Karanasios KA, Vasiliadou IA, Pavlou S, Vayenas DV. 2010. Hydrogenotrophic denitrification of potable water: A review. J Hazard Mater 180:20-37.

Lee KC, Rittmann BE. 2002. Applying a novel autohydrogenotrophic hollow-fiber membrane biofilm reactor for denitrification of drinking water. Water Res 36(8):2040-2052.

Levenspiel 0. 1980. The Monod equation: A revisit and a generalization to product inhibition situations. Biotechnol Bioeng 22(8):1671-1687.

Lu C, Gu P, He P, Zhang G, Song C. 2009. Characteristics of hydrogenotrophic denitrification in a combined system of gas-permeable membrane and a biofilm reactor. J Hazard Mater 168:1581-1589.

Martin KJ. 2013. Effect of Counter-diffusion, fluid dynamics, and biofilm morphology on membrane-supported biofilms. Notre Dame, Indiana: University of Notre Dame. p 288.

Martin KJ, Nerenberg R. 2012. The membrane biofilm reactor (MBfR) for water and wastewater treatment: Principles, applications, and recent developments. Bioresour Technol 122:83-94.

Martin KJ, Picioreanu C, Nerenberg R. 2013. Multidimensional modeling of biofilm development and fluid dynamics in a hydrogen-based membrane biofilm reactor (MBfR). Water Res 47(13):4739-4751. 
Matsumoto S, Terada A, Tsuneda S. 2007. Modeling of membrane-aerated biofilm: Effects of $\mathrm{C} / \mathrm{N}$ ratio, biofilm thickness and surface loading of oxygen on feasibility of simultaneous nitrification and denitrification. Biochem Eng J 37(1):98-107.

Morgenroth E, Wilderer PA. 2000. Influence of detachment mechanisms on competition in biofilms. Water Res 34(2):417-426.

Musvoto EV, Wentzel MC, Loewenthal RE, Ekama GA. 2000. Integrated chemicalphysical processes modelling-I. Development of a kinetic-based model for mixed weak acid/base systems. Water Res 34(6):1857-1867.

Nerenberg R, Rittmann BE. 2004. Hydrogen-based, hollow-fiber membrane biofilm reactor for reduction of perchlorate and other oxidized contaminants. Water Sci Technol 49(11-12):223-230.

Ontiveros-Valencia A, Tang Y, Krajmalnik-Brown R, Rittmann BE. 2013. Perchlorate reduction from a highly contaminated groundwater in the presence of sulfatereducing bacteria in a hydrogen-fed biofilm. Biotechnol Bioeng 110(12):3139-3147.

Ontiveros-Valencia A, Tang Y, Krajmalnik-Brown R, Rittmann BE. 2014. Managing the interactions between sulfate- and perchlorate-reducing bacteria when using hydrogen-fed biofilms to treat a groundwater with a high perchlorate concentration. Water Res 55:215-224.

Ontiveros-Valencia A, Ziv-el M, Zhao H-P, Feng L, Rittmann BE, Krajmalnik-Brown R. 2012. Interactions between nitrate-reducing and sulfate-reducing bactera coexisting in a hydrogen-fed biofilm. Environ Sci Technol 46:11289-11298.

Pavissich JP, Aybar M, Martin KJ, Nerenberg R. 2014. A methodology to assess the effects of biofilm roughness on substrate fluxes using image analysis, substrate profiling, and mathematical modelling. Water Sci Technol 69(9):1932-1941.

Picioreanu C, Kreft J-U, van Loosdrecht MCM. 2004. Particle-based multidimensional multispecies biofilm model. Appl Env Microb 70(5):3024-3040.

Picioreanu C, van Loosdrecht MCM, Curtis TP, Scott K. 2010. Model based evaluation of the effect of $\mathrm{pH}$ and electrode geometry on microbial fuel cell performance. Bioelectrochemistry 78(1):8-24.

Picioreanu C, van Loosdrecht MCM, Heijnen JJ. 2000. Two-dimensional model of bioiflm detachment caused by internal stress from liquid flow. Biotechnol Bioeng 72(2):205-218

Radu AI, Vrouwenvelder JS, Van Loosdrecht MCM, Picioreanu C. 2012. Effect of flow velocity, substrate concentration and hydraulic cleaning on biofouling of reverse osmosis feed channels. Chem Eng J 188:30-39.

Raskin L, Rittmann BE, Stahl DA. 1996. Competition and coexistence of sulfatereducing and methanogenic populations in anaerobic biofilms. Appl Env Microb 62(10):3847-3857.
Reichert P. 1994. A tool for simulation and data analysis of aquatic systems. Water Sci. Technol. 30(2):21-30.

Rittmann BE, McCarty PL. 2001. Environmental biotechnology. Madison, WI: McGraw Hill.

Sorensen J, Tiedje JM, Firestone RB. 1980. Inhibition by sulfide of nitric and nitrous oxide reduction by denitrifying Pseudomonas-fluorescens. Appl Env Microb 39(1):105-108.

Tang Y, Ontiveros-Valencia A, Feng L, Zhou C, Krajmalnik-Brown R, Rittmann BE. 2012. A biofilm model to understand the onset of sulfate reduction in denitrifying membrane biofilm reactors. Biotechnol Bioeng 110(3):763-772.

Tang Y, Ziv-el M, Zhou C, Shin JH, Ahn CH, Meyer K, Candelaria D, Friese D, Overstreet R, Scott R, et al. 2010. Bioreduction of nitrate in groundwater using a pilot-scale hydrogen-based membrane biofilm reactor. Front Environ Sci Engin China 4(3):280-285.

Terada A, Kaku S, Matsumoto S, Tsuneda S. 2006. Rapid autohydrogenotrophic denitrification by a membrane biofilm reactor equipped with a fibrous support around a gas-permeable membrane. Biochem Eng J 31(1):84-91.

Van Ginkel SW, Tang Y, Rittmann BE. 2011. Impact of precipitation on the treatment of real ion-exchange brine using the $\mathrm{H}_{2}$-based membrane biofilm reactor. Water Sci Technol 63(7):1453-1458.

Widdel F. 1988. Microbiology and ecology of sulfate- and sulfur-reducing bacteria. In: Zehnder AJB, editor. Biology of anaerobic microorganisms. New York, NY: John Wiley \& Sons.

Xia S, Zhang Y, Zhong F. 2009. A continuous stirred hydrogen-based polyvinyl chloride membrane biofilm reactor for the treatment of nitrate contaminated drinking water. Bioresour Technol 100:6223-6228.

Zhao H-P, Ontiveros-Valencia A, Tang Y, Kim B-0, VanGinkel S, Friese D, Overstreet R, Smith J, Evans P, Krajmalnik-Brown R, et al. 2014. Removal of multiple electron acceptors by pilot-scale, two-stage membrane biofilm reactors. Water Res 54:115-122.

Ziv-El MC, Rittmann BE. 2009. Water quality assessment of groundwater treated with a membrane biofilm reactor. Am Water Works Association J 101(12): 77-83.

\section{Supporting Information}

Additional Supporting Information may be found in the online version of this article at the publisher's web-site. 